\title{
Adverse Drug Reactions Among Patients Initiating Second-Line Antiretroviral Therapy in South Africa
}

\author{
Dorina Onoya $^{1}{ }^{1} \cdot$ Kamban Hirasen $^{1} \cdot$ Liudmyla van den Berg $^{2} \cdot$ Jacqui Miot $^{1} \cdot$ Lawrence C. Long $^{1,3}$. \\ Matthew P. Fox ${ }^{1,3,4}$
}

Published online: 24 July 2018

(c) The Author(s) 2018

\begin{abstract}
Introduction Understanding the occurrence of antiretroviral (ARV)-related adverse events (AEs) among patients receiving second-line antiretroviral therapy (ART) is important in preventing switches to more limited and expensive third-line regimens.

Objective This study aimed to estimate the rates and examine predictors of AEs among adult HIV-1-infected patients receiving second-line ART in the Right to Care (RTC) clinical cohort in South Africa.

Methods This was a cohort study of HIV-1-infected adult patients ( $\geq 18$ years of age) initiating standard second-line ART in South Africa from 1 April 2004 to 10 January 2016. Our primary outcome was the development of an AE within 24 months of initiating second-line therapy. We used Kaplan-Meier survival analysis to determine AE incidence in the first 24 months of second-line ART. Predictors of AEs were modelled using a Cox proportional hazards model.

Results A total of 7708 patients initiated second-line ART, with $44.5 \%$ developing at least one AE over the first 24 months of second-line treatment. The highest AE incidence was observed among patients receiving abacavir $(\mathrm{ABC})+$ lamivudine (3TC) + ritonavir-boosted lopinavir/atazanavir (LPVr/ATVr) (52.7/100 person-years (PYs), 95\% confidence interval (CI): 42.9-64.8), while patients initiated on a tenofovir (TDF) + emtricitabine (FTC)/3TC + LPVr regimen had the lowest rate of AEs (26.4/100 PYs, 95\% CI: 24.9-28.3). Clinical predictors of AEs included experiencing AEs when receiving first-line ART (adjusted hazard ratio (aHR) 2.3, 95\% CI: 1.9-2.8), lower CD4 cell count (0-199 vs. $\geq 350$ cells $/ \mathrm{mm}^{3}$; aHR 1.4, 95\% CI: 1.4-1.8), and switching to second-line therapy from an ABC-base first-line regimen (ABC+3TC + efavirenz/nevirapine [EFV/NVP] vs. TDF + 3TC/FTC + EFV/NVP; aHR 3.4, 95\% CI: 1.1-11.1).

Conclusions The rates of AEs were lowest among patients receiving a TDF-based second-line regimen. Patients with poorer health at the time of switch were at higher risk of AEs when receiving second-line ART and may require closer monitoring to improve the durability of second-line therapy.
\end{abstract}

Dorina Onoya

donoya@heroza.org

1 Health Economics and Epidemiology Research Office, Department of Internal Medicine, School of Clinical Medicine, Faculty of Health Sciences, University of the Witwatersrand, 39 Empire Road, Empire Park, Parktown, Johannesburg 2193, South Africa

2 Right to Care, Johannesburg, South Africa

3 Department of Global Health, Boston University School of Public Health, Boston, MA, USA

4 Department of Epidemiology, Boston University School of Public Health, Boston, MA, USA 


\section{Key Points}

Within 24 months of starting second-line therapy at a large HIV clinical cohort in South Africa, close to half of the patients $(3429 / 7708,44.5 \%)$ had experienced at least one second-line antiretroviral therapy-related adverse event (AE).

Rates of AEs among patients receiving secondline regimens such as $\mathrm{TDF}+\mathrm{FTC} / 3 \mathrm{TC}+\mathrm{LPVr}$ are lower than that of patients receiving other regimens $(3 \mathrm{TC}+\mathrm{ABC}+\mathrm{LPVr} / \mathrm{ATVr} ; \mathrm{AZT}+\mathrm{ddI}+\mathrm{LPVr}$; $\mathrm{AZT}+3 \mathrm{TC}+\mathrm{LPVr} / \mathrm{ATVr})$.

In resource-limited settings such as South Africa, where access to third-line therapy is not guaranteed, the early detection and effective management of AEs, including low-grade AEs, could improve patient outcomes in second-line therapy.

\section{Introduction}

Over the past 2 decades, expanded access to antiretroviral therapy (ART), in combination with more tolerable regimens, has shifted the impact of human immunodeficiency virus (HIV) infection from causing a fatal disease to a treatable chronic condition. South Africa is currently home to an estimated 7.2 million HIV-1-infected persons and has responded to the epidemic by enrolling $56 \%$ (approximately $3,831,730$ ) of HIV-1-infected patients receiving ART, by 2017 [1]. Furthermore, in 2017, approximately $38-45 \%$ of all HIV-1-infected persons receiving HIV treatment were virally suppressed $[2,3]$. This wide gap between the number of HIV-1-infected patients and those who are suppressed when receiving ART, as well as the growing number of persons entering the HIV treatment cascade, will eventually increase the demand for second-line therapy $[1,3,4]$. Currently, an estimated $17-25 \%$ of first-line ART patients in South Africa are expected to experience virologic failure (as defined by the World Health Organization [WHO]) within 5 years [5-8]. Moreover, data from the Right to Care (RTC) clinical cohort, one of the largest ART cohorts in the country, indicate that $>60 \%$ of patients not responding to first-line ART are switched to second-line therapy [9-11]. Therefore, it is essential to continuously monitor and appropriately respond to treatment failure risks to keep the ART programme effective and sustainable.

While various studies have quantified and examined the impact of adverse drug reactions (ADRs) among patients receiving first-line therapy, very few have looked at patients receiving second-line ART. In a systematic review examining the efficacy of treatment among ART-naive HIVinfected patients in Rome (Italy), early cessation of first-line ART (within 48 weeks of treatment initiation) was estimated at $25 \%$, with the most common reasons being by patients' decision (11\%), $8 \%$ due to ADRs (8\%), and 4\% due to virologic failure (4\%) [12]. We recently demonstrated that South African patients with possible ADRs receiving second-line ART were more likely (approximately $30 \%$ ) to experience treatment interruption within 2 years of second-line initiation [13]. While early treatment cessation has declined over the years, the removal of CD4 count eligibility criteria for starting ART will likely increase the number of asymptomatic individuals initiated on ART. These relatively healthy patients may be less tolerant of ART-associated ADRs, which may reverse the recent gains made in retention in HIV treatment. Although access to ART has significantly improved, patients in resource-limited settings (including South Africa) still have few HIV treatment options beyond second-line ART, hence the need to closely monitor ARTrelated ADRs among patients receiving second-line ART $[13,14]$. However, the growth of the South African ART programmes was not followed by an equivalent growth in adverse event (AE) monitoring systems, and routine clinical data on ART AEs offers, at best, indicators of ARTassociated ADRs.

This study aimed to use routine data to estimate the rate of, and examine predictors of, second-line ART-associated AEs (as a proxy for ADRs) in the first 24 months of secondline ART among adult HIV-1-infected patients in the RTC clinical cohort in South Africa.

\section{Methods}

\subsection{Study Design, Site and Population}

Using prospectively collected routine clinical data, we conducted a cohort study of adult ( $\geq 18$ years of age at second-line ART initiation) HIV-1-infected patients who initiated standard first-line ART (Table 1) and subsequently permanently switched to standard second-line ART (triple

Table 1 Standard first- and second-line antiretroviral therapy regimens in South Africa

\begin{tabular}{ll}
\hline Standard first-line regimens & Standard second-line regimens \\
\hline TDF +3TC/EFV + EFV/NVP & AZT/ABC-3TC-LPVr \\
AZT +3TC + EFV/NVP & TDF-3TC/FTC-LPVr \\
$\mathrm{d} 4 \mathrm{~T}+3 \mathrm{TC}+\mathrm{EFV} / \mathrm{NVP}$ & AZT/ABC-3TC-ATVr \\
$\mathrm{ABC}+3 \mathrm{TC}+\mathrm{EFV} / \mathrm{NVP}$ & TDF-3TC/FTC-ATVr \\
\hline
\end{tabular}

$T D F$ tenofovir, $3 T C$ lamivudine, $E F V$ efavirenz, $N V P$ nevirapine, $A Z T$ zidovudine, $d 4 T$ stavudine, $A B C$ abacavir, $L P V r$ lopinavir/ritonavir, $F T C$ emtricitabine, $A T V r$ atazanavir/ritonavir 
combination ART, including a standard protease inhibitor [PI]) between 1 April 2004 and 10 January 2016 within the RTC clinical cohort in South Africa [9, 15-20]. The analytic dataset included data from the date of second-line ART initiation (baseline) to 24 months post second-line ART initiation.

The RTC clinical cohort consists of 10 clinics (three nongovernmental organisation [NGO]-run clinics, four public Community Health Centres (CHCs) and three ART clinics embedded in public hospitals) located in the Gauteng and Mpumalanga provinces in South Africa [9]. The clinics were established or expanded as part of the public sector scale-up of ART in the country and receive technical support from RTC, an NGO that is funded by the United States Agency for International Development (USAID). HIV care and treatment within the RTC clinical cohort follows South African National ART guidelines [15-20]. During the study period, CD4 threshold for ART eligibility criteria was revised from $\leq 200$ cells $/ \mu \mathrm{L}$ in the 2004 guidelines to $\leq 350$ cells $/ \mu \mathrm{L}$ in the 2013 guideline update, and $\leq 500$ cells $/ \mu \mathrm{L}$ in 2015 . CD4 thresholds were removed from the 2016 HIV treatment policy [15-20]. Moreover, patients not responding to first-line ART (two consecutive viral loads $\geq 1000$ copies/mL within a 3-month period, with intensified adherence counselling administered between viral loads) are eligible to switch to second-line therapy [19]. Additionally, in the absence of virologic failure, the decision to switch a patient to secondline therapy may also be made based on diagnosed AEs/ resistance to first-line drugs.

Clinical data, including laboratory data from the National Health Laboratory Services (NHLS), from the ART clinics were captured on site and stored in an electronic patient management system, TherapyEdge-HIVTM [9].

\subsection{Analytic Variables}

\subsubsection{Adverse Events (AEs) in the Initial 24 Months of Second-Line Antiretroviral Therapy (ART)}

In the absence of reliable pharmacovigilance ADR data, we use second-line ART-related AEs as proxy measures. Therefore, the primary outcome was the first occurrence of any one of the following ART-related conditions during medical visits, up to 24 months after second-line ART initiation: dyslipidaemia, dermatitis/skin conditions neuropathy, diarrhoea/nausea/vomiting, gynaecomastia/breast conditions, hepatitis, lactic acidosis, decreased kidney function, anaemia, depression, and sleep disorders/insomnia. These conditions have previously been identified as possible drug reactions to both first- and second-line ARVs [21].

These conditions were ascertained by abstraction from clinical visit notes. However, in addition to clinical notes, anaemia episodes were also identified using haemoglobin
( $\mathrm{Hb}$ ) measurements defined as $\mathrm{Hb}<13.0 \mathrm{~g} / \mathrm{dL}$ in men and $<12.0 \mathrm{~g} / \mathrm{dL}$ in women. Similarly, episodes of renal insufficiencies while receiving second-line ART were also identified using estimated glomerular filtration rate (eGFR) measures. Renal problems were defined as eGFR $\leq 59 \mathrm{~mL} /$ $\min / 1.73 \mathrm{~m}^{2}$ for both males and females.

AEs were also identified up to 6 months before the switch to second-line ART to allow adjustments for unresolved AEs that occurred while receiving first-line ART. AEs occurring while receiving second-line ART were distinguished from those occurring while receiving first-line therapy by the respective start dates of each AE. In addition to the first occurrence of an AE, a measure of the frequency of AEs up to 24 months after the switch to second-line ART was determined.

\subsubsection{Baseline Explanatory Variables}

We defined baseline as the time of initiating second-line ART, and indicators were considered baseline if they were taken up to 3 months before the initiation of second-line ART. Baseline data included (1) demographic variables; (2) clinical and laboratory variables (e.g. WHO stage, body mass index [BMI], CD4 count and viral load); and (3) treatment variables (e.g. ART regimen, and treatment start and stop date). WHO staging information is generally interpreted as asymptomatic, mildly symptomatic, moderately symptomatic, and severely symptomatic. BMI (measured in $\mathrm{kg} / \mathrm{m}^{2}$ ) was categorised as being underweight $(\mathrm{BMI}<18.5)$, normal $(18.5 \leq \mathrm{BMI}<25)$, overweight $(25 \leq \mathrm{BMI}<30)$, and obese (BMI $\geq 30$ ). Additionally, we included a variable summarising the CD4 response when receiving first-line ART, defined as the change in CD4 count from first-line ART initiation to the date of switching to second-line ART.

\subsection{Follow-Up Time}

Person-time accrued from the date of second-line ART initiation until the outcome of interest, completion of 24 months of second-line ART, or the last date seen at the clinic during the first year of second-line ART (for those who died, were lost to follow-up [LTFU], or transferred out).

\subsection{Statistical Analysis}

Data analysis was conducted using STATA version 14 (StataCorp LLC, College Station, TX, USA). Baseline demographic and clinical characteristics were presented using medians and interquartile ranges (IQRs) for continuous variables, while categorical variables were described using percentages. Kaplan-Meier survival analysis was used to determine the incidence of AEs in the first 24 months of second-line ART. Predictors of experiencing an AE (first 
reported AE within 24 months of second-line initiation) were modelled using complete case analysis with the use of a Cox proportional hazards model. Models were adjusted for baseline demographic and clinical characteristics. Additionally, we looked at the frequency of AEs by the initial second-line ART regimens.

Variables with a $p$ value $<0.05$ in crude analyses were entered in the multivariate model. Schoenfeld residuals were used to test the assumption of proportional hazards. Interaction terms with time-varying covariates were created for variables that violated the proportional hazards assumption. Variables were excluded from the multivariate model when the inclusion of the interaction term did not resolve the proportional hazards assumption violation, except for the initiating second-line regimen, in which case the model was stratified.

\section{Results}

\subsection{Cohort Description and Baseline Characteristics}

Table 2 presents the demographic and clinical characteristics of the study cohort at second-line ART initiation $(N=7708)$. Nearly two-thirds of the cohort were female $(65.0 \%)$, and the median age at second-line initiation was 37.4 years (IQR 32.3-43.9). The median CD4 cell count at second-line ART initiation was 212 cells/ $\mu \mathrm{L}$ (IQR 101-344), with $47.2 \%$ of patients switching to second-line therapy at a CD4 cell count of $<200$ cells/ $\mu \mathrm{L}$. The majority of patients were switched to second-line ART with viral loads $\geq 1000$ copies $/ \mathrm{mL}(82.9 \%)$. Nearly half of all patients had a normal BMI (47.8\%), while close to two-thirds of patients were at WHO stage I (62.8\%) at the time of switching to secondline ART.

At the time of second-line ART initiation, $52.7 \%$ of patients had been receiving first-line ART for 2 or more years. Overall, $42.6 \%$ of patients initiated second-line ART on a regimen of tenofovir (TDF) + emtricitabine (FTC)/lamivudine (3TC) + ritonavir-boosted lopinavir (LPVr), 34.9\% were prescribed zidovudine (AZT) $+3 \mathrm{TC}+\mathrm{LPVr} / \mathrm{ritona}-$ vir-boosted atazanavir (ATVr), and $19.5 \%$ were prescribed AZT + didanosine (ddI) + LPVr. Only $3.1 \%$ were switched to $3 \mathrm{TC}+$ abacavir $(\mathrm{ABC})+\mathrm{LPVr}$.

\subsection{AEs in the First 24 Months of Second-Line ART}

Overall, 3429 (44.5\%) patients experienced an AE in the first 24 months of second-line treatment (Table 3). The overall rate of AEs while receiving second-line ART was 36.7/100 person-years (PYs; 95\% confidence interval [CI] 35.3-38.1). This was highest among patients receiving a regimen including ABC (52.7/100 PYs, 95\% CI 42.9-64.8) or ddI (51.2/100
PYs, 95\% CI 47.5-55.2), while patients switched to a TDF(26.5/100 PYs, 95\% CI 24.9-28.3) or AZT-based secondline regimen had the lowest AE rates (42.8/100 PYs, 95\% CI 40.2-45.5). Table 3 presents the first AE experienced by the initial second-line ART regimen, up to 24 months of second-line ART.

Anaemia was the most common first AE experienced, with 2389 first AE cases (31.0\% prevalence and $68.0 \%$ of all AEs); $78.5 \%$ of these anaemia episodes were mild $(24.0 \%$ overall), $16.9 \%$ were moderate (5\% overall), and $4.6 \%$ were severe (1.0\% overall) (Fig. 1). Nearly half (43.6\%) of all first anaemia cases were among patients receiving $\mathrm{AZT}+3 \mathrm{TC}+\mathrm{LPVr} / \mathrm{ATVr}$, while the next most common $\mathrm{AE}$ was decreased kidney function (264 cases, or 3\% overall), among which $90.9 \%$ were mild, $6.3 \%$ were moderate and $2.8 \%$ were severe cases. Kidney problems were less likely among patients receiving $\mathrm{TDF}+\mathrm{FTC} / 3 \mathrm{TC}+\mathrm{LPVr}$ (5.0\% overall) compared with patients receiving $\mathrm{ABC}+3 \mathrm{TC}+\mathrm{LPVr} / \mathrm{ATVr}(12.0 \%)$. After anaemia $(35.0 \%$ overall), patients receiving $\mathrm{AZT}+\mathrm{ddI}+\mathrm{LPVr}$ also experienced gastrointestinal conditions $(6.0 \%)$ and dyslipidaemia $(4.0 \%)$.

\subsection{Predictors of AEs in the First 24 Months of Second-Line ART}

Table 4 presents the crude (hazard ratio [HR]) and adjusted HR (aHR) estimates of experiencing an $\mathrm{AE}$ in the first 24 months of second-line ART. In adjusted analyses, when compared with patients initiating secondline ART of TDF + FTC/3TC + LPVr, patients receiving $\mathrm{AZT}+\mathrm{ddI}+\mathrm{LPVr}$ (aHR 1.5, 95\% CI 1.2-1.9) and $\mathrm{AZT}+3 \mathrm{TC}+\mathrm{LPVr} / \mathrm{ATVr}$ (aHR $1.3,95 \%$ CI 1.1-1.1) were more likely to experience AEs while receiving second-line ART.

In the adjusted model including the total cohort, patients who had experienced an $\mathrm{AE}$ in the last 6 months of firstline ART were twice more likely to experience an AE in the first 24 months of second-line ART (aHR 2.3, 95\% CI 1.9-2.8). Male patients had a $43 \%$ reduced risk compared with females (aHR 0.6, 95\% CI 0.5-0.7), and patients who switched to second-line ART at 40 years of age or older (HR1.4, 95\% CI 1.0-1.8) were more likely to experience AEs compared with those who were younger (18-30 years) at the switch.

Patients with a lower CD4 cell count at baseline were $38.0 \%$ more likely to experience AEs ( $0-199$ vs. $\geq 350$ cells/ $\mu \mathrm{L}$ : aHR 1.4, 95\% CI 1.1-1.8). Patients who were underweight at second-line ART initiation had a higher risk of AEs (underweight vs. normal-weight patients: aHR 1.4, 95\% CI 1.2-1.7), whereas obese and overweight patients had a reduced likelihood of AEs (overweight vs. normal BMI: 
Table 2 Demographic and clinical characteristics of patients initiating second-line antiretroviral therapy from 1 April 2004 to 10 January 2016

\begin{tabular}{|c|c|}
\hline Variables & Total $[N=7708]$ \\
\hline \multicolumn{2}{|l|}{ Sex at second-line initiation } \\
\hline Female & $5010(65.0)$ \\
\hline Male & $2698(35.0)$ \\
\hline \multicolumn{2}{|c|}{ Age at second-line initiation, years } \\
\hline Median (IQR) & $37.4(32.3-43.9)$ \\
\hline $18-29$ & $1220(15.8)$ \\
\hline $30-39$ & $3489(45.3)$ \\
\hline$\geq 40$ & 2999 (38.9) \\
\hline \multicolumn{2}{|c|}{ CD4 cell count at second-line initiation, cells $/ \mathrm{mm}^{3}$} \\
\hline Median (IQR) & $212(101-344)$ \\
\hline 0-199 & $1436(47.2)$ \\
\hline $200-349$ & $864(28.4)$ \\
\hline$\geq 350$ & $742(24.4)$ \\
\hline \multicolumn{2}{|c|}{ Viral load at second-line initiation, copies $/ \mathrm{mL}$} \\
\hline Median (IQR) & $11,841(2100-60,784)$ \\
\hline$<1000$ & $941(17.0)$ \\
\hline $1000-9999$ & $1686(30.5)$ \\
\hline$\geq 10,000$ & $2896(52.4)$ \\
\hline \multicolumn{2}{|l|}{ BMI at second-line initiation } \\
\hline Underweight & $440(7.8)$ \\
\hline Normal & $2681(47.8)$ \\
\hline Overweight & $1530(27.3)$ \\
\hline Obese & $959(17.1)$ \\
\hline \multicolumn{2}{|c|}{ WHO stage at second-line initiation } \\
\hline I & $2580(62.8)$ \\
\hline II & $582(14.2)$ \\
\hline III/IV & $944(23.0)$ \\
\hline \multicolumn{2}{|c|}{ Time on first-line ART before the switch, months } \\
\hline Median (IQR) & $25.5(13.8-44.8)$ \\
\hline $0-12$ & $1564(20.3)$ \\
\hline $12-24$ & $2086(27.1)$ \\
\hline$\geq 24$ & $4058(52.7)$ \\
\hline \multicolumn{2}{|c|}{ Regimen at second-line initiation } \\
\hline $3 \mathrm{TC}+\mathrm{ABC}+\mathrm{LPVr}$ & $238(3.1)$ \\
\hline $\mathrm{AZT}+3 \mathrm{TC}+\mathrm{LPVr} / \mathrm{ATVr}$ & $2686(34.9)$ \\
\hline $\mathrm{AZT}+\mathrm{ddI}+\mathrm{LPVr}$ & $1501(19.5)$ \\
\hline $\mathrm{TDF}+\mathrm{FTC} / 3 \mathrm{TC}+\mathrm{LPVr}$ & $3283(42.6)$ \\
\hline \multicolumn{2}{|l|}{ Year of second-line initiation } \\
\hline 2004-2009 & 1895 (24.6) \\
\hline 2010-2012 & $3618(46.9)$ \\
\hline 2013-2016 & $2195(28.5)$ \\
\hline \multicolumn{2}{|c|}{ Non-ARV comedication at second-line initiation } \\
\hline No & $4752(61.7)$ \\
\hline Yes & $2956(38.4)$ \\
\hline \multicolumn{2}{|c|}{ *Time (days) to first AE in the first 24 months of second-line ART } \\
\hline Median (IQR) & $84(28-237)$ \\
\hline $0-90$ & $1810(52.8)$ \\
\hline $91-180$ & $547(16.0)$ \\
\hline$\geq 181$ & $1072(31.3)$ \\
\hline
\end{tabular}


Table 2 (continued)

\begin{tabular}{lc}
\hline Variables & Total $[N=7708]$ \\
\hline Time (days) to first AE in the first 24 months of second-line ART by initial second-line regimen [medium \\
(IQR) ${ }^{*}$ \\
$\mathrm{ABC}+3 \mathrm{TC}+\mathrm{LPVr} / \mathrm{ATVr}$ & $57(5-155)$ \\
$\mathrm{AZT}+3 \mathrm{TC}+\mathrm{LPVr} / \mathrm{ATVr}$ & $56(26-181)$ \\
$\mathrm{AZT}+\mathrm{ddI}+\mathrm{LPVr}$ & $85(28-224)$ \\
$\mathrm{TDF}+\mathrm{FTC} / 3 \mathrm{TC}+\mathrm{LPVr}$ & $134(28-308)$ \\
\hline
\end{tabular}

Data are expressed as $n(\%)$ unless otherwise specified

$I Q R$ interquartile range, $B M I$ body mass index, $W H O$ World Health Organization, $A R T$ antiretroviral therapy, $A R V$ antiretroviral, $3 T C$ lamivudine, $A B C$ abacavir, $L P V r$ lopinavir/ritonavir, $A T V r$ atazanavir/ritonavir, $A Z T$ zidovudine, $d d I$ didanosine, $T D F$ tenofovir, $F T C$ emtricitabine, $A E$ adverse event

Table 3 Details of AEs, drug substitutions and treatment interruptions among patients with incident AEs up to 24 months of second-line initiation

\begin{tabular}{|c|c|c|c|c|}
\hline \multicolumn{4}{|c|}{ Initial second-line ART regimen } & \multirow[t]{2}{*}{ Total } \\
\hline $\begin{array}{l}3 \mathrm{TC}+\mathrm{ABC}+\mathrm{LPVr} / \\
\mathrm{ATVr}\end{array}$ & $\begin{array}{l}\mathrm{AZT}+3 \mathrm{TC}+\mathrm{LPVr} / \\
\mathrm{ATVr}\end{array}$ & $\mathrm{AZT}+\mathrm{ddI}+\mathrm{LPVr}$ & $\begin{array}{l}\mathrm{TDF}+\mathrm{FTC} / 3 \mathrm{TC}+ \\
\mathrm{LPVr}\end{array}$ & \\
\hline$n(\operatorname{col} \%)$ & $n(\operatorname{col} \%)$ & $n(\operatorname{col} \%)$ & $n(\operatorname{col} \%)$ & $n(\operatorname{col} \%)$ \\
\hline$[N=117]$ & {$[N=1298]$} & {$[N=822]$} & {$[N=1192]$} & {$[N=3429]$} \\
\hline \multicolumn{5}{|l|}{ d } \\
\hline $80(68.4)$ & $1015(78.2)$ & $524(63.6)$ & $711(59.7)$ & $2330(68.0)$ \\
\hline $28(23.9)$ & $77(5.9)$ & $8(1.0)$ & $151(12.7)$ & $264(7.7)$ \\
\hline $2(1.7)$ & $68(5.2)$ & $60(7.3)$ & $115(9.7)$ & $245(7.1)$ \\
\hline $2(1.7)$ & $52(4.0)$ & $83(10.1)$ & $82(6.9)$ & $219(6.4)$ \\
\hline $2(1.7)$ & $28(2.2)$ & $44(5.4)$ & $52(4.4)$ & $126(3.7)$ \\
\hline $3(2.6)$ & $29(2.2)$ & $49(6.0)$ & $40(3.4)$ & $121(3.5)$ \\
\hline - & $29(2.2)$ & $54(6.6)$ & $41(3.4)$ & $124(3.6)$ \\
\hline
\end{tabular}

First $\mathrm{AE}$ in the observation period

$\begin{array}{llc}\text { Anaemia } & 80(68.4) & 1015(78.2) \\ \text { Decreased kidney function } & 28(23.9) & 77(5.9) \\ \text { Dyslipidaemia } & 2(1.7) & 68(5.2) \\ \text { Gastrointestinal conditions } & 2(1.7) & 52(4.0) \\ \text { Neuropathy } & 2(1.7) & 28(2.2) \\ \text { Skin conditions } & 3(2.6) & 29(2.2) \\ \text { Others } & - & 29(2.2)\end{array}$

Frequency of AE episodes in the initial 24 months of second-line ART

\begin{tabular}{|c|c|c|c|c|c|}
\hline One & $39(33.3)$ & $594(45.8)$ & $342(41.6)$ & $681(57.1)$ & $1656(48.3)$ \\
\hline Two & $20(17.1)$ & $335(25.8)$ & $209(25.4)$ & $264(22.2)$ & $828(24.2)$ \\
\hline Three or more & $58(49.6)$ & $369(28.4)$ & $271(33.0)$ & $247(20.7)$ & $945(27.6)$ \\
\hline \multicolumn{6}{|c|}{ rug substitutions on second-line ART (24 months) } \\
\hline None & $177(74.4)$ & $2097(78.1)$ & $885(59.0)$ & $2608(79.4)$ & $5767(74.8)$ \\
\hline One & $38(16.0)$ & $345(12.8)$ & $446(29.7)$ & $471(14.4)$ & $1300(16.9)$ \\
\hline Two & $12(5.0)$ & $171(6.4)$ & $134(8.9)$ & $154(4.7)$ & $471(6.1)$ \\
\hline Three or more & $11(4.6)$ & $70(2.6)$ & $36(2.4)$ & $50(1.5)$ & $167(2.2)$ \\
\hline \multicolumn{6}{|c|}{ reatment interruptions on second-line ART ( 24 months) } \\
\hline None & $216(90.8)$ & $2491(92.7)$ & $1273(84.8)$ & $3019(92.0)$ & $6999(90.8)$ \\
\hline One & $19(8.0)$ & $180(6.7)$ & $205(13.7)$ & $243(7.4)$ & $647(8.4)$ \\
\hline Two & $2(0.8)$ & $13(0.5)$ & $22(1.5)$ & $17(0.5)$ & $54(0.7)$ \\
\hline Three or more & $1(0.4)$ & $2(0.1)$ & $1(0.1)$ & $4(0.1)$ & $8(0.1)$ \\
\hline
\end{tabular}

$A E s$ adverse events, $A R T$ antiretroviral therapy, $3 T C$ lamivudine, $A B C$ abacavir, $L P V r$ lopinavir/ritonavir, $A T V r$ atazanavir/ritonavir, $A Z T$ zidovudine, $d d l$ didanosine, $T D F$ tenofovir, FTC emtricitabine

aHR 0.8, 95\% CI 0.6-1.0; and obese vs. normal BMI: aHR $0.8,95 \%$ CI $0.6-1.0)$.

In stratified models, similar results were observed for having a history of AEs before switching to second-line ART, sex, age (in patients receiving a ddI-based regimen),
WHO stage, baseline CD4 count (in patients receiving a ddI-based regimen) and BMI. Among patients who were initially switched to TDF + FTC/3TC + LPVr, a CD4 decline after first-line ART initiation was predictive of 


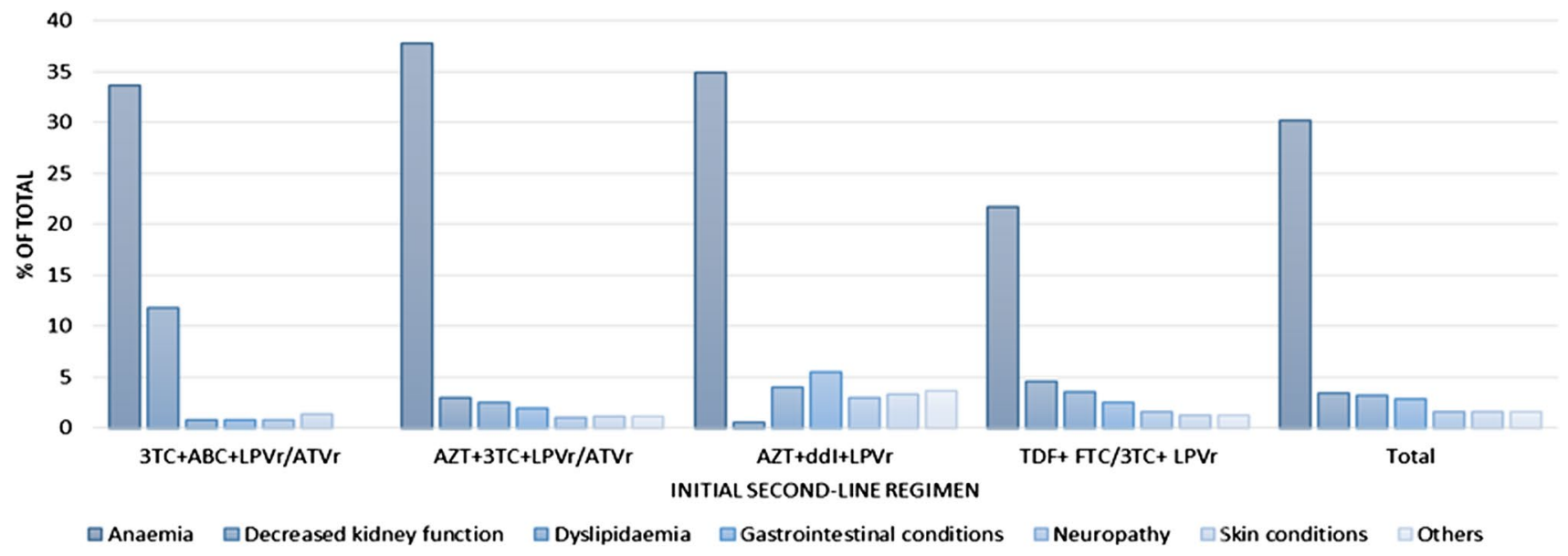

Fig. 1 Proportion of incident adverse events in initial 24 months of second-line ART among HIV-1 infected patients in South Africa

AEs while receiving second-line ART (CD4 decrease $\geq 50$ cells $/ \mu \mathrm{L}$ vs. no CD4 change: aHR $2.5,95 \%$ CI 1.3-5.0).

\subsection{Predictors of Experiencing Two or More AEs Among Patients Who Had Incident AEs in the First 24 Months of Second-Line ART}

In logistic regression analyses, predictors of having two or more AEs among patients who had incident AEs (Table 4) were having a history of AEs while receiving first-line ART (adjusted odds ratio [aOR] 2.3, 95\% CI 1.8-2.9), having a baseline CD4 count $<199$ cells/ $\mu \mathrm{L}$ compared with those with CD4 $\geq 350$ cells/ $\mu \mathrm{L}$ (aOR 1.6, 95\% CI 1.0-2.4), starting second-line ART with AZT + 3TC + LPVr/ATV (aOR 1.7, 95\% CI 1.2-2.3) or AZT + ddI + LPVr (aOR 2.0, 95\% CI 1.4-2.8) compared with those who started on $\mathrm{TDF}+\mathrm{FTC} / 3 \mathrm{TC}+\mathrm{LPVr}$. Additionally, although imprecise, the risk of multiple $\mathrm{AE}$ episodes was higher among patients who had switched from $\mathrm{ABC}+3 \mathrm{TC}+$ efavirenz/nevirapine (EFV/NVP) [aOR 10.8, 95\% CI 1.2-94.0] compared with patients who switched from TDF + 3TC/FTC + EFV/NVP. Compared with women, male patients were less likely to have had multiple AEs in the first 2 years of second-line ART (aOR 0.7, 95\% CI 0.5-0.9).

\section{Discussion}

We aimed to determine the rates and examine clinical predictors of developing an ART-related AE in the first 24 months of second-line ART among HIV-1-infected adult patients within the RTC clinical cohort. Overall, $44.5 \%$ of patients experienced an $\mathrm{AE}$ in the 24 months of observation, across second-line ART regimens. This proportion is higher than the $19 \%$ observed in a Malawi cohort (12 months follow-up)
[22] and 22.3\% from a 2011 cross-sectional study (including children) in Uganda [23]. The variations of AE rates for patients receiving second-line ART highlight the inconsistent AE reporting practices and follow-up times across settings and studies [24].

Similar to other sub-Saharan African populations, we found that the rates of AEs were higher among patients receiving a second-line regimen that included AZT and ddI compared with a TDF-based regimen [25, 26]. The first AE was mainly anaemia, more so among patients who started second-line ART with an AZT-containing regimen. Despite the differing study methods, a cross-sectional study among patients receiving second-line ART in Malawi found similar anaemia levels, i.e. 33.2 versus $31.0 \%$ in our study [27].

The second most common first AEs were kidney problems, affecting 3\% of the sample. While kidney problems were expected to occur mainly among patients receiving TDF, they were more frequent among patients who were switched to an ABC-based second-line regimen [21,28]. This difference in the occurrence of kidney problems among patients switched to ABC for second-line ART possibly stems from TDF contraindications or pre-existing kidney problems at the time of the switch [29]. Patients who started second-line ART on an ABC-based regimen were also at considerably higher risk of frequent AEs in the first 2 years of second-line ART. Furthermore, patients who experienced AEs in the last 6 months of first-line ART were more susceptible to multiple AEs after the switch to second-line ART. Additional predictors of AEs among patients receiving second-line ART include low baseline BMI and CD4 count. The relationship between CD4 cell count and the development of AEs is unclear in recent literature, with both higher and lower CD4 cell counts having been associated with incident AEs [30, 31]. However, the results suggest that patients with poor health 


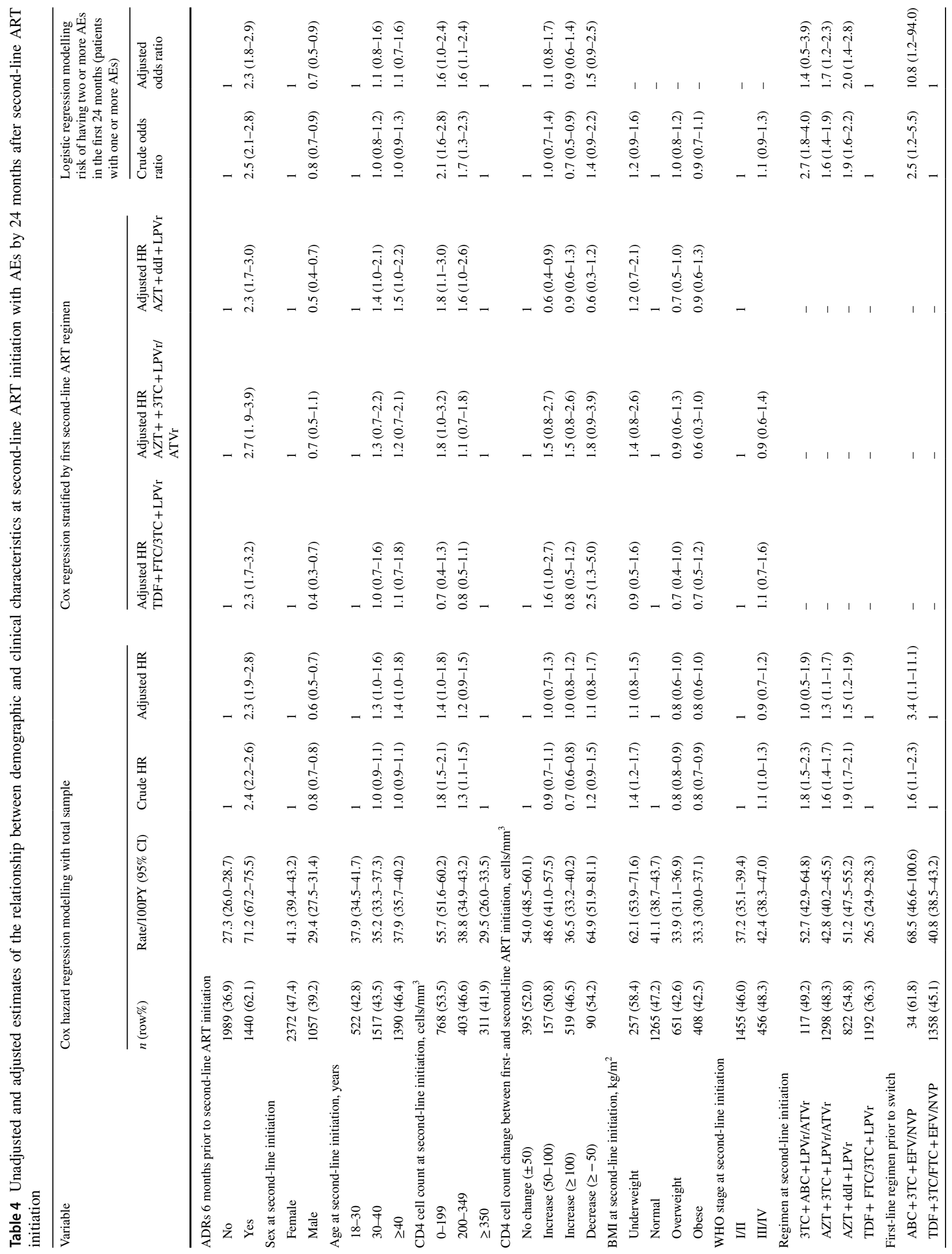


who switch to second-line ART may struggle to clear preexisting conditions, are less able to cope with the challenge with new drugs, and require closer monitoring.

Demographic factors associated with an increased risk of AEs while receiving second-line ART were older age at second-line ART initiation ( $\geq 40$ vs. 18-30 years) and being female. Similar to our findings, a gender difference in ART discontinuation and AE pattern has been previously observed [32], with women being more likely to develop peripheral neuropathy, particularly at low $\mathrm{Hb}$ levels [33-35].

\section{Limitations}

The interpretation of these results is limited to the context from which participants were drawn. The RTC cohort consisted of patients from health facilities in Gauteng and Mpumalanga province and may not be representative of patients across South Africa. Due to the lack of formal AE reporting systems in South Africa, AE rates may be underestimated [36-38], and more systematic and structured pharmacovigilance systems are needed to improve the accuracy of reported AEs. While rates of AEs were lower among patients receiving a TDF-based regimen, $36.3 \%$ over 2 years is not negligible. Further studies with more extended follow-up periods are needed to assess the longer-term implication of AEs and the potential fluidity in predictors of such events.

\section{Conclusions}

TDF in second-line ART is least associated with incident and frequent AEs in the first 24 months of second-line ART. However, patients with poor health indicators at switch, those with a history of AEs while receiving first-line ART, and those with TDF contraindications are at a considerably higher risk of incident AEs when receiving secondline ART, and need closer monitoring and support in order to thrive. Furthermore, the size of the ART programme in South Africa, coupled with high rates of comorbidities, such as tuberculosis, warrants active pharmacovigilance to obtain data on actual ADRs to better monitor the second-line ART programme.

Acknowledgements The authors gratefully acknowledge the directors and staff of the study sites, as well as Right to Care, the Non-Governmental Organization supporting the study sites through a partnership of the South African National and Gauteng provincial Department of Health with the USAID. Most of all, the authors thank the patients attending the clinics for their continued trust in the treatment provided at the clinic. 
Author contributions DO, KH and MPF conceptualised the analysis, analysed the data and drafted the manuscript. LCL, JM and LvdB assisted in the analysis and contributed to the interpretation of the results, as well as manuscript preparation.

\section{Compliance with Ethical Standards}

Conflict of interest Dorina Onoya, Kamban Hirasen, Liudmyla van den Berg, Jacqui Miot, Lawrence C. Long and Matthew P. Fox have no conflicts of interest that are directly relevant to the content of this study.

Funding This work was supported by the generous American people through the USAID under the terms of Cooperative Agreement 674-A12-00020 to RTC; INROADS USAID-674-A-12-00029 to the Health Economics and Epidemiology Research Unit and to Boston University. The contents of this paper are the responsibility of the authors and do not necessarily reflect the views of the funding agencies or participating clinics or patients.

Ethical Approval All data were fully anonymised for analyses. Ethics approval for the retrospective data review was obtained from the Human Research Ethics Committee of the University of Witwatersrand (M140201) and the Boston University Institutional Review Board (H-29768).

Patient Consent Following Sect. 3 of the recommendations regarding the provisions for waiver or alteration of the informed consent requirements under the South African Department of Health and Human Services (HHS) Regulations at 45 CFR 46.116(d) and the Declaration of Helsinki, a waiver for individual patient consent was obtained.

Open Access This article is distributed under the terms of the Creative Commons Attribution-NonCommercial 4.0 International License (http://creativecommons.org/licenses/by-nc/4.0/), which permits any noncommercial use, distribution, and reproduction in any medium, provided you give appropriate credit to the original author(s) and the source, provide a link to the Creative Commons license, and indicate if changes were made.

\section{References}

1. National Department of Health (South Africa). National Department of Health annual report 2016/2017. Pretoria: National Department of Health; 2017.

2. UNAIDS. Global AIDS update 2017. UNAIDS; 2017.

3. Johnson LF, Dorrington RE, Moolla H. Progress towards the 2020 targets for HIV diagnosis and antiretroviral treatment in South Africa. S Afr J HIV Med. 2017;18(1):a694.

4. Estill J, et al. The need for second-line antiretroviral therapy in adults in sub-Saharan Africa up to 2030: a mathematical modelling study. Lancet HIV. 2016;3(3):e132-9.

5. Boulle A, Van Cutsem G, Hilderbrand K, Cragg C, Abrahams $\mathrm{M}$, Mathee S, et al. Seven-year experience of a primary care antiretroviral treatment programme in Khayelitsha, South Africa. Aids. 2010;24(4):563-72.

6. Fox MP, Van Cutsem G, Giddy J, Maskew M, Keiser O, Prozesky $\mathrm{H}$, et al. Rates and predictors of failure of first-line antiretroviral therapy and switch to second-line ART in South Africa. J Acquir Immune Defic Syndr. 2012;60(4):428.

7. Nglazi MD, Lawn SD, Kaplan R, Kranzer K, Orrell C, Wood $\mathrm{R}$, et al. Changes in programmatic outcomes during 7 years of scale-up at a community-based antiretroviral treatment service in South Africa. J Acquir Immune Defic Syndr. 2011;56(1):e1.

8. World Health Organization (WHO). Consolidated guidelines on the use of antiretroviral drugs for treating and preventing HIV infection: recommendations for a public health approach. 2nd ed. Geneva: WHO Press; 2016.

9. Fox MP, Maskew M, Brennan AT, Evans D, Onoya D, Malete G, et al. Cohort profile: the right to care clinical HIV cohort, South Africa. BMJ Open. 2017;7(6):e015620.

10. Fox MP, Maskew M, MacPhail AP, Long L, Brennan AT, Westreich D, et al. Cohort profile: the Themba lethu clinical cohort, Johannesburg, South Africa. Int J Epidemiol. 2012;42(2):430-9.

11. Sanne IM, Westreich D, Macphail AP, Rubel D, Majuba P, Rie A. Long-term outcomes of antiretroviral therapy in a large HIV/ AIDS care clinic in urban South Africa: a prospective cohort study. J Int AIDS Soc. 2009;12(1):38.

12. Prosperi MC, Fabbiani M, Fanti I, Zaccarelli M, Colafigli M, Mondi A, et al. Predictors of first-line antiretroviral therapy discontinuation due to drug-related adverse events in HIVinfected patients: a retrospective cohort study. BMC Infect Dis. 2012;12(1):296.

13. Onoya D, Brennan AT, Berhanu R, Berg L, Buthelezi T, Fox MP. Changes in second-line regimen durability and continuity of care in relation to national ART guideline changes in South Africa. J Int AIDS Soc. 2016;19(1):20675.

14. Onoya D, Nattey C, Budgell E, van den Berg L, Maskew M, Evans D, et al. Predicting the need for third-line antiretroviral therapy by identifying patients at high risk for failing secondline antiretroviral therapy in South Africa. AIDS Patient Care STDs. 2017;31(5):205-12.

15. National Department of Health (South Africa). National antiretroviral treatment guidelines 2010. Pretoria: National Department of Health; 2010.

16. Department of Health (South Africa). National antiretroviral treatment guidelines 2004. Pretoria: National Department of Health; 2004.

17. Department of Health (South Africa). Circular on new criteria for initiating adults on ART at CD4 count of 350 cells $/ \mathrm{ml}$ and below 2011. Pretoria: National Department of Health; 2011.

18. Department of Health (South Africa). National antiretroviral treatment guidelines 2013. Pretoria: National Department of Health; 2013.

19. Department of Health (South Africa). National consolidated guidelines-for the prevention of mother-to-child transmission of HIV (PMTCT) and the management of HIV in children, adolescents and adults 2015. Pretoria: National Department of Health; 2015.

20. Department of Health (South Africa). Implementation of the universal test and treat strategy for HIV positive patients and differentiated care for stable patients. Pretoria: National Department of Health; 2016.

21. Orrell C. Antiretroviral adverse drug reactions and their management: how to recognise, manage and avoid adverse effects of antiretrovirals. Continuing Medical Education. 2011;29(6):234-7.

22. Hosseinipour M, Kumwenda J, Weigel R, Brown L, Mzinganjira $\mathrm{D}$, Mhango B, et al. Second-line treatment in the Malawi antiretroviral programme: high early mortality, but good outcomes in survivors, despite extensive drug resistance at baseline. HIV Med. 2010;11(8):510-8.

23. Namukanja PMM. Adverse effects on second-line highly active antiretroviral therapy (HAART) among HIV infected adults and children treated at Mildmay Uganda. Pretoria: University of Limpopo (Medunsa Campus); 2011. http://ulspace.ul.ac.za/handl e/10386/532. 
24. Miller V (ed). ARV drugs, adverse events, case definition, grading, laboratory diagnosis and treatment monitoring. Presentations at the 2nd interest meeting, Feb 28-29 in Geneva, Switzerland; 2010. http://www.hivforum.org/tox-a-aes/59-arv-drugs-adverseevents-case-definition-grading-laboratory-diagnosis-and-treat ment-monitoring.

25. Ciaffi L, Koulla-Shiro S, Sawadogo A, le Moing V, EymardDuvernay S, Izard S, et al. Efficacy and safety of three second-line antiretroviral regimens in HIV-infected patients in Africa. AIDS. 2015;29(12):1473.

26. Spaulding A, Rutherford GW, Siegfried N. Tenofovir or zidovudine in three-drug combination therapy with one nucleoside reverse transcriptase inhibitor and one non-nucleoside reverse transcriptase inhibitor for initial treatment of HIV infection in antiretroviral-naïve individuals. Cochrane Database Syst Rev. 2010;10:008740.

27. Ngongondo M, Rosenberg NE, Stanley CC, et al. Anemia in people on second line antiretroviral treatment in Lilongwe, Malawi: a cross-sectional study. BMC Infect Dis. 2018;18:39.

28. Kearney BP, Mathias A, Mittan A, Sayre J, Ebrahimi R, Cheng AK. Pharmacokinetics and safety of tenofovir disoproxil fumarate on coadministration with lopinavir/ritonavir. J Acquir Immune Defic Syndr. 2006;43(3):278-83.

29. Evans D, Maskew M, Heneger C, Sanne I. Estimated use of abacavir among adults and children enrolled in public sector antiretroviral therapy programmes in Gauteng Province, South Africa. South Afr J HIV Med. 2012;13(3):134-7.

30. Kesselring AM, Wit FW, Sabin CA, Lundgren JD, Gill MJ, Gatell $\mathrm{JM}$, et al. Risk factors for treatment-limiting toxicities in patients starting nevirapine-containing antiretroviral therapy. Aids. 2009;23(13):1689-99.

31. Lichtenstein KA, Armon C, Buchacz K, Chmiel JS, Moorman $\mathrm{AC}$, Wood $\mathrm{KC}$, et al. Initiation of antiretroviral therapy at CD4 cell counts $\geq 350$ cells $/ \mathrm{mm}^{3}$ does not increase incidence or risk of peripheral neuropathy, anemia, or renal insufficiency. J Acquir Immune Defic Syndr. 2008;47(1):27-35.

32. Kempf M-C, Pisu M, Dumcheva A, Westfall AO, Kilby JM, Saag MS. Gender differences in discontinuation of antiretroviral treatment regimens. J Acquir Immune Defic Syndr. 2009;52(3):336-41.

33. Mehta SA, Ahmed A, Laverty M, Holzman RS, Valentine F, Sivapalasingam S. Sex differences in the incidence of peripheral neuropathy among kenyans initiating antiretroviral therapy. Clin Infect Dis. 2011;53(5):490-6.

34. Arenas-Pinto A, Thompson J, Musoro G, Musana H, Lugemwa A, Kambugu A, et al. Peripheral neuropathy in HIV patients in sub-Saharan Africa failing first-line therapy and the response to second-line ART in the EARNEST trial. J NeuroVirol. 2016;22(1):104-13.

35. Volberding PA, Levine AM, Dieterich D, Mildvan D, Mitsuyasu R, Saag M. Anemia in HIV infection: clinical impact and evidence-based management strategies. Clin Infect Dis. 2004;38(10):1454-63.

36. Agu KA, Isah MA, Oqua D, Habeeb MA, Agada PO, Ohiaeri SI, et al. Incidence of adverse drug reactions in patients on antiretroviral therapy: a study of pharmaceutical care in HIV interventions in Nigeria. West Afr J Pharm. 2013;24(1):30-42.

37. Mehta UC. Pharmacovigilance: the devastating consequences of not thinking about adverse drug reactions. CME. 2011;29(6):247-51.

38. Kiguba R, Karamagi C, Waako P, Ndagije HB, Bird SM. Recognition and reporting of suspected adverse drug reactions by surveyed healthcare professionals in Uganda: key determinants. BMJ Open. 2014;4(11):e005869. 